\section{A rare case of scrofuloderma along with lupus vulgaris}

\author{
Chiara Sabbadini, ${ }^{1}$ Julia Oberschmied, ${ }^{1}$ \\ Martina Tauber, ${ }^{2}$ Carla Nobile ${ }^{1}$ \\ ${ }^{1}$ Department of Dermatology, \\ Venereology and Allergology, Hospital \\ Brunico, Brunico; ${ }^{2}$ Department of \\ Pathology, Central Hospital Bolzano, \\ Bolzano, Italy
}

\begin{abstract}
Cutaneous forms of tuberculosis are rare, comprising about $1-1.5 \%$ of all cases, and show a wide range of clinical manifestations. Here we present a case of a patient with left cervical ulcerated lymphadenopathy associated with a violaceous plaque in the area of the manubrium of sternum. We performed a biopsy of the plaque for histopathology, a polymerase chain reaction (PCR) to test for mycobacteria and a smear of the ulcerated lymph node. Histopathology results showed a dermal infiltrate consisting of epithelioid granulomas without necrosis, PCR was negative, and the culture was positive for M. tuberculosis. We made the diagnosis of scrofuloderma associated with lupus vulgaris. The patient was treated with an anti-tuberculous therapy with clinical regression of the lesions. Our case emphasizes the importance of recognizing that tuberculosis can occur as a primary cutaneous pathology, with a challenging diagnosis that requires the correlation of clinical findings with diagnostic testing.

Transient acantholytic dermatosis (TAD) is a relatively common entity that has been also noted to occur in patients with cancer. Herein, we describe a case of transient acantholytic dermatosis occurring in a patient with a history of prostate cancer status post radiation, now being treated with combination therapy with pembrolizumab and carboplatin-pemetrexed for advanced lung adenocarcinoma. Our case emphasizes the importance of being cognizant of TAD and its associations, particularly in cancer patients.
\end{abstract}

\section{Introduction}

Tuberculosis (TB) is one of the top 10 causes of death in the world and the leading cause from a single pathogenic agent. Millions of humans continue to fall sick with tuberculosis every year. ${ }^{1}$ Cutaneous tuberculosis (CTB) is a rare clinical manifestation, comprising $1-1.5 \%$ of all extrapulmonary manifestations of tuberculosis. $^{2}$ The classification of CTB shows a high complexity. A classification in two primary categories is widely accepted: true CTB and tuberculids. ${ }^{3}$ It is defined true CTB if Mycobacterium tuberculosis can be detected at lesional sites with common testing tools like smear, culture or PCR examination. It can be classified into two sub-groups according to their cause: inoculation from an exogenous source (which results from the entry of mycobacteria into the skin) or from an endogenous source. Endogenous TB is due to direct spread or auto-inoculation and includes scrofuloderma, orificial TB and some cases of lupus vulgaris. Lupus vulgaris, tuberculous gumma, and acute miliary TB are caused by hematogenous transmission. ${ }^{3}$ Important factors to determine the clinical presentation of CTB include the pathogenicity of the organism, the portal of infection, the immune status of the host and other different local factors in the skin like relative vascularity, trauma, lymphatic drainage and proximity to lymph nodes. ${ }^{4}$ Due to the fact that the clinical manifestations are varied and complicated (mimic diverse skin conditions), it is a great challenge to make the right diagnosis for the dermatologists in daily practice. ${ }^{3}$ There are only few cases in literature describing associated forms of CTB. ${ }^{5}$ To our knowledge, we present the third case of scrofuloderma associated with lupus vulgaris.

\section{Case Report}

We report a case of a 57-year-old Indian man, who had a 2-year history of a cervical lymphadenopathy, followed by the Department of Pneumology. He lived in Italy since a few years, had no relevant medical history and didn't take any drugs. In February 2018 he performed a cervical lymph node biopsy. The histopathologic findings showed extensive lymphohistiocytic inflammatory reaction with a central abscess and a peripheral granulomatous reaction, the PCR amplification was positive for atypical mycobacteria. QuantiFERON-TB Gold (QFT-G) test was borderline. The patient refused the purposed antibiotic treatment because of non-compliance. In January 2020 he was admitted to our dermatological department with a 5-month history of cervical wound healing disorder. On physical examination, he had mild, tender left anterior cervical lymphadenopathy with two hard nodular erythematous formations associated with a violaceous plaque with satellite papules and ulceration in the area of
Correspondence: Chiara Sabbadini, Hospital Brunico, Department of Dermatology, Venereology and Allergology, Via Ospedale 11, 39031 Brunico, Italy.

Tel.: +39.0474581230.

E-mail: chiara.sabbadini@sabes.it

Key words: Cutaneous tuberculosis, Scrofuloderma, Lupus vulgaris.

Contributions: The authors contributed equally.

Conflict of interest: The authors declare no potential conflict of interest.

Funding: None.

Ethical approval: Not applicable.

Consent for publication: Written informed consent was obtained from the patient for publication of this case report and any accompanying images.

Availability of data and material: Data and materials are available by the authors.

Please cite this article as: Sabbadini C, Oberschmied J, Tauber M, Nobile C. A rare case of scrofuloderma along with lupus vulgaris. Dermatol Rep 2021;13:8993.

Received for publication: 20 October 2020.

Revision received: 16 December 2020.

Accepted for publication: 7 January 2021.

This work is licensed under a Creative Commons Attribution-NonCommercial 4.0 International License (CC BY-NC 4.0).

COCopyright: the Author(s), 2021

Licensee PAGEPress, Italy

Dermatology Reports 2021; 13:8993

doi:10.4081/dr.2021.8993

the manubrium of sternum (Figure 1).

One of the cervical lymph nodes ulcerated after 1 week and evolved with the persistence of a secreting fistula with drainage of serous-purulent content. We performed a blood test, a smear from the ulcerated area of the neck and a skin biopsy from the plaque. In our skin biopsy, the histopathologic findings showed a dermal infiltrate consisting of epithelioid granulomas without necrosis surrounded by lymphocytes and multinucleate giant cells (Figure 2).

The PCR amplification was negative for mycobacteria. The histologic features were compatible with lupus vulgaris. The culture of material obtained from the cervical draining lesion was positive for Mycobacterium tuberculosis. QuantiFERON-TB Gold test was positive, laboratory findings were within normal 
range, HIV test was negative. We also performed a chest radiography and a chest, neck and abdomen computed tomography which excluded visceral tuberculosis. A diagnosis of scrofuloderma associated with lupus vulgaris was made. For the planning of the correct treatment and management, the pneumologists were involved. We started an antituberculous quadruple therapy combining rifampicin $600 \mathrm{mg} /$ daily, isoniazid $300 \mathrm{mg} /$ daily, ethambutol 1.2 $\mathrm{g}$ /daily, and pyrazinamide $2 \mathrm{~g}$ /daily. At the dermatologic follow up visit, after 2 months of therapy, cutaneous lesions were regressed, leaving hyperpigmented patches. The treatment is still ongoing with only rifampicin $600 \mathrm{mg} /$ daily and isoniazid 300 $\mathrm{mg} /$ daily.

\section{Discussion}

Lupus vulgaris is the most common type of CTB, as showed in a recent Spanish study, representing $61.11 \%$ of the cases. The disease can either be acquired endogenously, by contiguous spread, haematogenous or lymphatic dissemination, or exogenously by direct inoculation. Lupus vulgaris sometimes develops on cervical scars of scrofuloderma that had occurred previously, usually in childhood. ${ }^{6,7}$ Clinically, lesions are generally reddish-brown, solitary and sharply defined. Clinical variations exist and are defined as classic plaque, hypertrophic ulcerative and vegetating. ${ }^{8}$ It occurs in previously sensitized individuals with high immunity. ${ }^{?}$ Without therapy, its course usually extends over many years, leading to significant impairment of function and disfiguration. ${ }^{9}$ Scrofuloderma emerges from direct invasion of the bacilli into the skin from an underlying tuberculous process, most of the time tuberculous lymphadenitis and tuberculosis of the epididymis, bone and joints. ${ }^{7,9}$ Lesions present as firm, painless, subcutaneous, red-brown nodules overlying an infected focus, which gradually enlarge and suppurate forming ulcers and sinus tracts that drain watery, purulent, or caseous material. ${ }^{8}$

Immunopathologically, initial immune response to Mycobacterium tuberculosis consists in an adaptive immune response engaging Th1 cells (CD4+ T-cells) and macrophages. If the mycobacteria survive a second stage begins, in which macrophages induce the production of cytokines resulting in the recruitment and activation of monocytes, lymphocytes, neutrophils and dendritic cells. Cellular activation and interleukin liberation stimulate macrophages to differentiate into epithelioid and giant cells that will organize themselves into granulomas according to individual host factors. ${ }^{10}$ Cutaneous TB is recognized to form a continuous immunopathologic spectrum, ranging from a high intensity of cell-mediated immunity, for example in lupus vulgaris, to a low intensity of cellmediated immunity, like in scrofuloderma. ${ }^{11}$ Rarely, as showed in our case report, these findings can be associated. In our opinion, this association is linked to the long course of the untreated lymph node disease. In the English published works, we found only two other combined case reports, which are

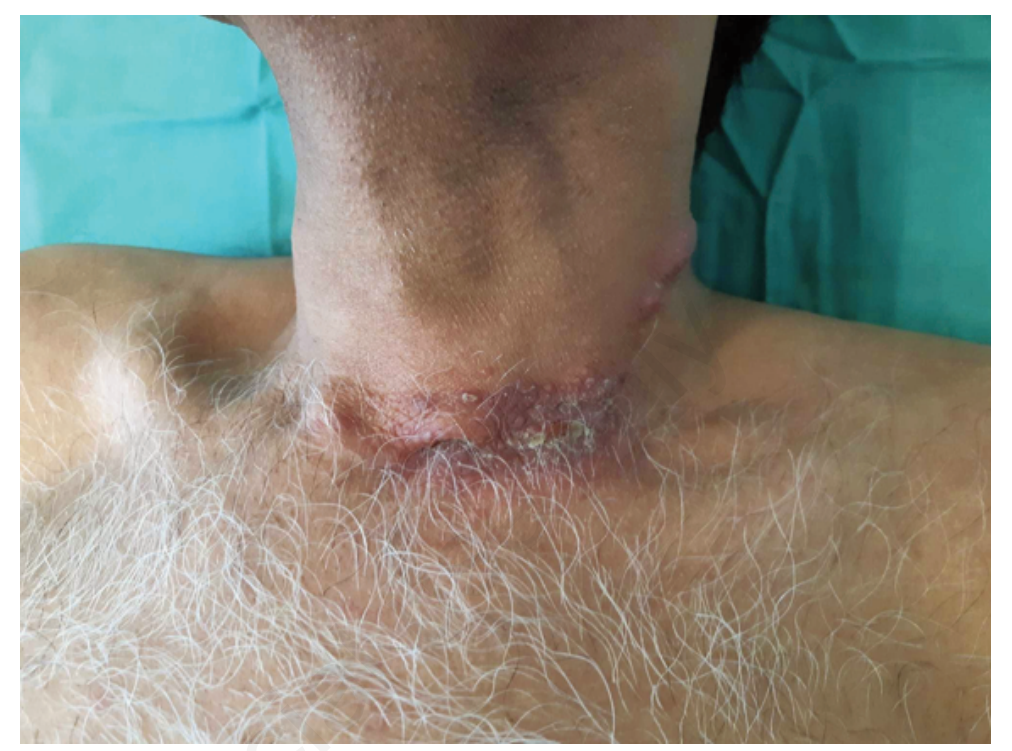

Figure 1. Patient at presentation. Left cervical lymphadenopathy associated with a violaceous plaque with satellite papules and ulceration in the area of the manubrium sternum.

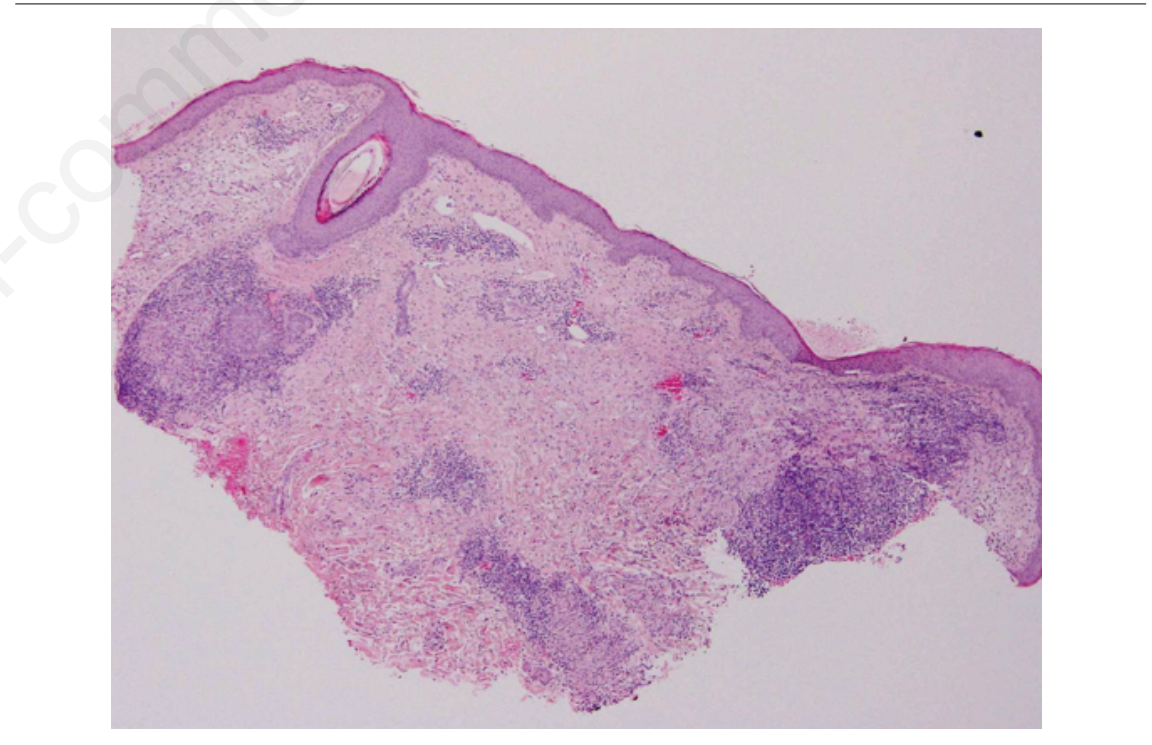

Figure 2. Dermal infiltrate consisting of epithelioid granulomas without necrosis surrounded by lymphocytes and multinucleate giant cells (Haematoxylin and Eosin stain, 4x).

Table 1. Cases of scrofuloderma associated with lupus vulgaris.

Article type Age Gender Localivation Clinical presentation

\begin{tabular}{lllllr} 
Alia ao lu et al. (2006) ${ }^{9}$ & Case report & 70 & F & Neck & Ulcerous-discharging abscess on the neck, giant atrophic plaque on the chest \\
Tuli et al. (2014) & Case report & 27 & M & Neck & Two ulcers associated with an erythematous, psoriasiform scaling plaque \\
\hline
\end{tabular}


summarized in Table $1.9^{9,12}$ To our knowledge, here we present the third case.

Histopathology is usually necessary for an early diagnosis of CTB, and with PCR even the smallest tissue sample can be analyzed and amplified for mycobacterial DNA sequences, confirming its presence. Our laboratory uses for PCR amplification the insertion sequence IS 6110 because of the repetitive nature of this element in the mycobacterial genome. A generic criticism of this technique lies in its sensitivity; some nontuberculous mycobacteria have multiple copies of sequences that are homologous to IS6110 and may thus hybridize with the IS6110 probe. $^{13}$ This could explain the initial PCR positivity in our patient for atypical mycobacteria which may have led to inappropriate treatment. Despite these limitations, the IS6110-RFLP (restriction fragment length polymorphism) method remains one of the most used approaches for Mycobacterium tuberculosis typing and was long considered the gold standard technique in the molecular epidemiological investigations of TB. ${ }^{14}$ Mycobacterial culture remains the most reliable method to determine the presence of mycobacteria and their sensitivities, but the yield is often low and takes many weeks. ${ }^{8}$ In our case, the culture was positive after about five weeks. Nonetheless, a combination of diagnostic tests is needed to confirm a diagnosis when a case of CTB is suspected.

Tuberculosis has a strong relationship with human immunodeficiency virus (HIV) infection. Therefore, testing for a HIV infection is recommended for all patients. ${ }^{4}$ Skin tuberculosis can be a sign of possible visceral tuberculous infection, which may indicate a poor prognosis, and patients can therefore benefit from early diagnosis and treatment. ${ }^{6}$ Chemotherapy is the treatment of choice with the aim to cure the disease as rapidly as possible to prevent relapses and the development of resistance. The recommended treatment consists of two phases: an initial bactericidal or intensive phase for the rapid destruction of a large population of mycobacterium and for the resolution of the symptoms, after which the patients are no longer infectious, followed by a continuation or sterilizing phase. ${ }^{4}$ The initial phase includes a daily dose of isoniazid, rifampicin, pyrazinamide and ethambutol given for a total of 2 months, followed by two drugs (isoniazid and rifampicin) for 4 months. ${ }^{2,4,15}$ The long duration of the treatment could negatively influence the patient's compliance. Patients who do not adhere to the prescribed treatment are likely to become drug resistant. A recommended method in order to address these problems is prescribing fixed-drug combination products. ${ }^{4}$ The ending of the therapy is defined more accurately by the total number of doses taken than by the length of treatment. Consequently, the response to the therapy must be assessed clinically. Based on the fact that viable mycobacteria can still be cultured from clinically cured lesions, treatment should be continued for at least 2 months after complete involution of the lesions. ${ }^{4}$

\section{Conclusions}

Cutaneous TB is an uncommon form of tuberculosis, difficult to diagnose due to its rare nature and the fact that it may present in different clinical forms. Therefore, it is challenging for the dermatologist to find the right diagnosis. Cutaneous TB requires the correlation of clinical findings with diagnostic testing. There are only a few cases in literature which describe scrofuloderma along with lupus vulgaris. Chemotherapy is still the treatment of choice and the response should be assessed clinically.

\section{References}

1. World Health Organization. Global tuberculosis report 2018. Geneva, Switzerland: World Health Organization; 2018.

https://apps.who.int/iris/handle/10665/2 74453.

2. van Zyl L, du Plessis J, Viljoen J. Cutaneous tuberculosis overview and current treatment regimens. Tuberc Edinb Scotl 2015;95:629-38.

3. Chen Q, Chen W, Hao F. Cutaneous tuberculosis: A great imitator. Clin Dermatol 2019;37:192-9.

4. Handog EB, Gabriel TG, Pineda RTV. Management of cutaneous tuberculosis. Dermatol Ther 2008;21:154-61.

5. Mann D, Sant'Anna FM, Schmaltz CAS, et al. Cutaneous tuberculosis in Rio de Janeiro, Brazil: description of a series of 75 cases. Int $J$ Dermatol 2019;58:1451-9.

6. Marcoval J, Alcaide F. Evolution of cutaneous tuberculosis over the past 30 years in a tertiary hospital on the European Mediterranean coast. Clin Exp Dermatol 2013;38:131-6.

7. Motta A, Feliciani C, Toto P, et al. Lupus vulgaris developing at the site of misdiagnosed scrofuloderma. J Eur Acad Dermatol Venereol 2003;17:313-35.

8. Frankel A, Penrose C, Emer J. Cutaneous tuberculosis: a practical case report and review for the dermatologist. J Clin Aesthetic Dermatol 2009;2:19-27.

9. Aliağaoğlu C, Atasoy M, Karakuzu A, et al. Rapidly developing giant sized lupus vulgaris on the chest associated with bilateral scrofuloderma on the neck. J Dermatol 2006;33:481-5.

10. Santos JBD, Figueiredo AR, Ferraz CE, et al. Cutaneous tuberculosis: epidemiologic, etiopathogenic and clinical aspects - part I. An Bras Dermatol 2014;89:219-28.

11. Sehgal VN. Cutaneous tuberculosis. Dermatol Clin 1994;12:645-53.

12. Tuli IP, Verma S, Thaku BK, Joshi D. Lupus vulgaris associated with Scrofuloderma. Sahel Med J 2014;17: 34-6.

13. McHugh TD, Newport LE, Gillespie SH. IS6110 homologs are present in multiple copies in mycobacteria other than tuberculosis-causing mycobacteria. J Clin Microbiol 1997;35:1769-71.

14. Jagielski $\mathrm{T}$, van Ingen $\mathrm{J}$, Rastogi $\mathrm{N}$, et al. Current Methods in the Molecular Typing of Mycobacterium tuberculosis and Other Mycobacteria. Biomed Res Int 2014:645802.

15. Furin J, Cox H, Pai M. Tuberculosis. Lancet 2019;393:1642-56. 